ANNALES

UNIVERSITATIS MARIAE CURIE-SKŁODOWSKA

LUBLIN - POLONIA

VOL. LXIX, 1-2

SECTIO AA

2014

\title{
Hydroxyapatite catalyzed cyclization of aryl enones: Solvent-free efficient synthesis of some 4-aryl-5,6-dihydro- 6-(substituted phenyl)-4H-1,3-oxazine-2-amines
}

\author{
G. Thirunarayanan \\ Department of Chemistry, Annamalai University, \\ Annamalainagar-608002, India. \\ e-mail:drgtnarayanan@gmail.com
}

Some 4-aryl-5,6-dihydro-6-(substituted phenyl)-4H-1,3-oxazine-2-amine derivatives including 4-(4-methyl-1-naphthyl)-5,6dihydro-6-(substituted phenyl)-4H-1,3-oxazine-2-amines have been synthesised by hydroxyapatite catalyzed solvent-free cyclization of aryl chalcones and urea using microwave irradiation under solventfree condition. The yields of the oxazines were more than $85 \%$. The synthesised oxazine amines have been characterized by their physical constants, analytical and spectroscopic data.

Key words: Oxazine-2-amines, Enones, Hydroxyapatite, Solventfree synthesis.

\section{INTRODUCTION}

The Oxazine or unsaturated oxazine derivatives are important bioactive six membered heterocyclic compounds which possess one oxygen and one nitrogen atom $[1,2]$. Depend upon the relative position of these tow atoms and the double bond, these molecule exists many isomeric structures such as 1,2 or 1,3 or 1, 4 oxazines [3]. These oxazines were medicinally important due to the presence of oxygen, nitrogen heteroatoms along with a double bonds in their structural moieties [4]. The important medicinal activities of these oxazines are anti-bacterial, 
anti-fungal [4-6], anti-plasmodial [7], anti-cancer [8], anti-depressants [9], cytotoxicity [10], anti-osteoplastic [11], anti- tumour [12], anti-oxidant [13], anti-tuberculosis [14], anti-neoplastic[15], antagonists [16], antiinflammatory [17], anti-infectants [18], IKB kinase beta [19] and PTP-1B inhibition [20]. These oxazine derivatives were applied for improving the super resolution Microscope [21], synthesis of eosinophils [22], identification and separation of neutrophils [23]. Many oxazine derivatives were used as a dyes [24]. Now-a-days scientists, organic chemists are interested for solvent-free synthesis [4, 25, 26, 27]. Numerous solvent assisted and solvent-free synthetic methods were available for synthesis of oxazine derivatives [28-31]. Hetero Diels-alder reaction [4], ring closure [32], Betti base induced condensation [27], Mannich type condensation-cyclization [5] and cyclization of chalcones [6] were used for synthesis of oxazine derivatives. Verma et. al. have synthesised some benzoxazine/oxazine fused isoquinolines and naphthyridines by solvent-free method [5]. Elarfi and Al-difar have synthesised some 1,3-oxazine derivatives by solvent-assisted method from chalcones and urea[6]. More than 75\% yield of dihydro-2H-benzoand naphtho-1, 3-oxazine derivatives were prepared by Mathew et al.[5] using eco-friendly method. Efficient synthesis of some 1, 3-oxazine-4thiones were synthesised by $\mathrm{N}$-methyl imidazole promoted solvent-free conditions. Sapkal et al., have studied the role of ammonium acetate for solvent-free synthesis of 1,3-disubstituted-2,3-dihydro- ${ }^{1} \mathrm{H}$-naphyl oxazines [27]. Within the above view, there is no information available in the literature for the solvent-free synthesis of 4-aryl-5,6-dihydro-6(substituted phenyl)-4H-1,3-oxazine-2-amines derivatives including 4-(4methyl-1-naphthyl)-6-(substituted phenyl)-4H-1, 3-oxazine-2-amines by cyclization of enones. Therefore the author have taken effort to synthesize some aryl oxazines including 4-(4-methyl-1-naphthyl)-6-(substituted phenyl) 4H-1,3-oxazine-2-amines by cyclization of enones under solvent-free conditions.

\section{EXPERIMENTAL}

\subsection{General}

All chemicals were used in this study were purchased from SigmaAldrich and Merck Chemical companies. Mettler FP51 melting point apparatus was used for determining the melting point of all synthesized oxazine-2-amines in open glass capillaries and are uncorrected. The 
AVATAR-300 Fourier transform spectrophotometer was used for recording infrared spectra $\left(\mathrm{KBr}, 4000-400 \mathrm{~cm}^{-1}\right)$ of all oxazines in $\mathrm{KBr}$ disc. The Bruker AV400 series type NMR spectrometer was utilized for recording NMR spectra of all oxazines, operating at $400 \mathrm{MHz}$ for ${ }^{1} \mathrm{H}$ and $100 \mathrm{MHz}$ for ${ }^{13} \mathrm{C}$ spectra in $\mathrm{CDCl}_{3}$ solvent using TMS as internal standard. Mass spectra of all synthesised oxazines were recorded on SHIMADZU mass spectrometer using chemical ionization technique.

\subsection{Preparation of hydroxyapatite}

Hydroxyapatite catalyst was prepared by aqueous precipitation method reported in literature [33]. Suspension $\left(10 \mathrm{~cm}^{3}\right)$ of $0.5 \mathrm{M}$ calcium hydroxide was prepared from calcium hydroxide powder. The degased suspension was stirred vigorously and heated at $40^{\circ} \mathrm{C}$ for $2 \mathrm{~h}$. About $10 \mathrm{~cm}^{3}$ of phosphoric acid solution $(0.3 \mathrm{M})$ was added drop-wise to it and kept aside an hour. The $\mathrm{pH}$ of the solution was adjusted to 9.5 by adding ammonium hydroxide solution. The mixture was stirred using magnetic stirrer for $2 \mathrm{~h}$ at the speed of $25 \mathrm{rpm}$, aged for $24 \mathrm{~h}$ in room and centrifuged to completion of precipitation. The precipitate was dried at $100^{\circ} \mathrm{C}$ for $12 \mathrm{~h}$. The obtained calcium hydroxyapatite catalyst have $1.8 \mathrm{Ca} / \mathrm{P}$ ratio was kept in vacuum desiccator.

\subsection{Preparation of chalcones}

The aryl chalcones were prepared according to literature procedure [31]. An appropriate equimolar quantities of aryl methyl ketones ( $2 \mathrm{mmol})$, various substituted benzaldehydes $(2 \mathrm{mmol})$ in methanol $\left(10 \mathrm{~cm}^{3}\right)$ and $1 \mathrm{~g}$ hydroxyapatite was added and the mixture was stirred at room temperature for $5 \mathrm{~min}$. Methanol was evaporated to give a homogeneous solid. About $5 \mathrm{~cm}^{3}$ of water was added to this solid and the mixture was irradiated by microwave for the appropriate time. After completion of reaction, dichloromethane $\left(20 \mathrm{~cm}^{3}\right)$ was added, followed by simple filtration. The solution was concentrated and to purified by recrystallization. The purities of synthesized chalcones were examined by their physical constants, IR, ${ }^{1} \mathrm{H}$ and ${ }^{13} \mathrm{C}$ NMR and Mass spectral data reported in literature[31]. 
2.4. Synthesis of 4-(aryl)-5,6-dihydro-6-(substituted phenyl)-4H-1,3-oxazine-2-amines

An appropriate equi-molar quantities of chalcones ( $2 \mathrm{mmol})$, urea $(2 \mathrm{mmol})$ and $0.2 \mathrm{~g}$ of hydroxyapatite were taken in a $50 \mathrm{~cm}^{3}$ beaker, closed with the lid. This mixture was subjected to microwave irradiation for 2-4 minutes at 650W (Scheme 1) (Samsung, Microwave Oven, $100-700 \mathrm{~W})$. After completion of the reaction, dichloromethane $\left(20 \mathrm{~cm}^{3}\right)$ was added, followed by simple filtration. The solution was concentrated and purified by re-crystallization. The synthesized oxazines were characterized by their physical constants, and mass fragments were presented in Table 1.<smiles>[R]C=CC=[PH2+]</smiles>
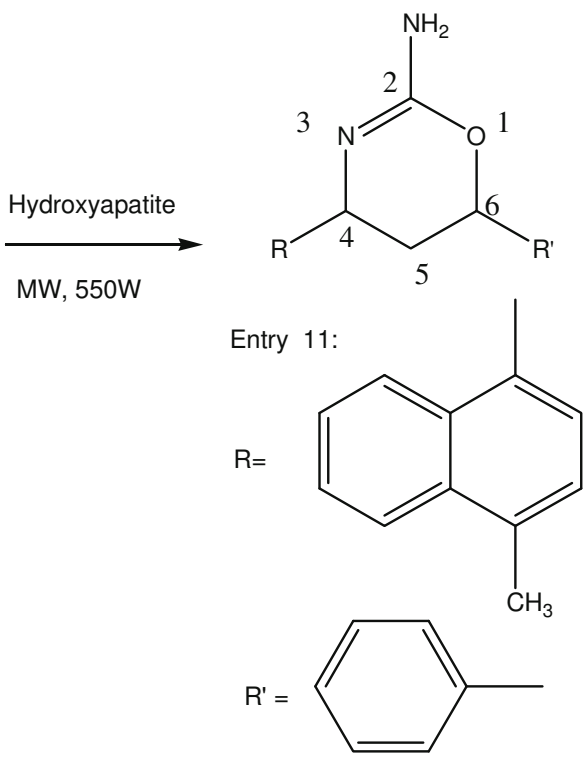

Scheme 1. Synthesis of 4-aryl-5,6-dihydro-6-(substituted phenyl)-4H-1,3-oxazine-2-amines hydroxyapatite catalyzed cyclization of aryl chalcones and urea under microwave irradiation. 


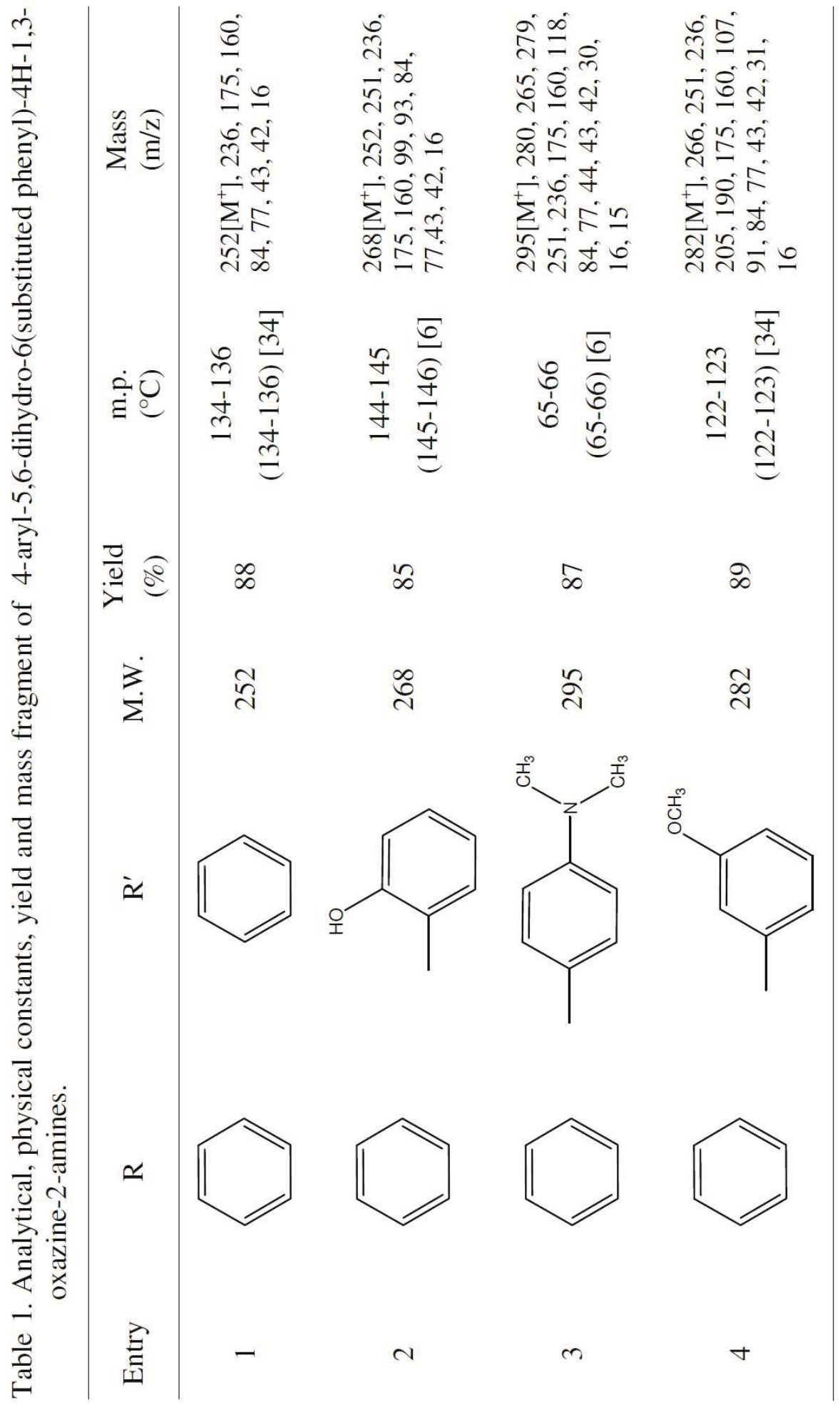




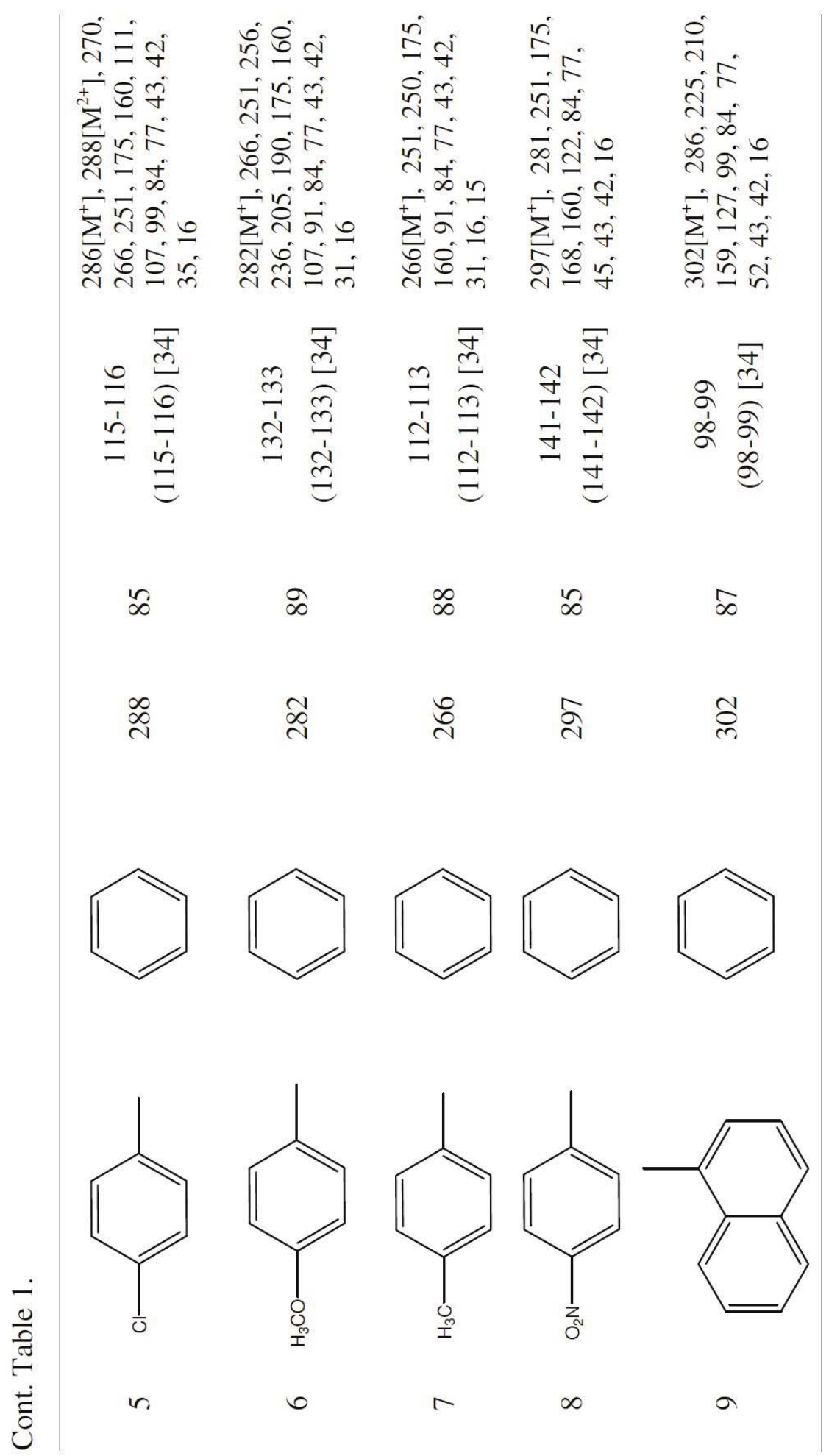




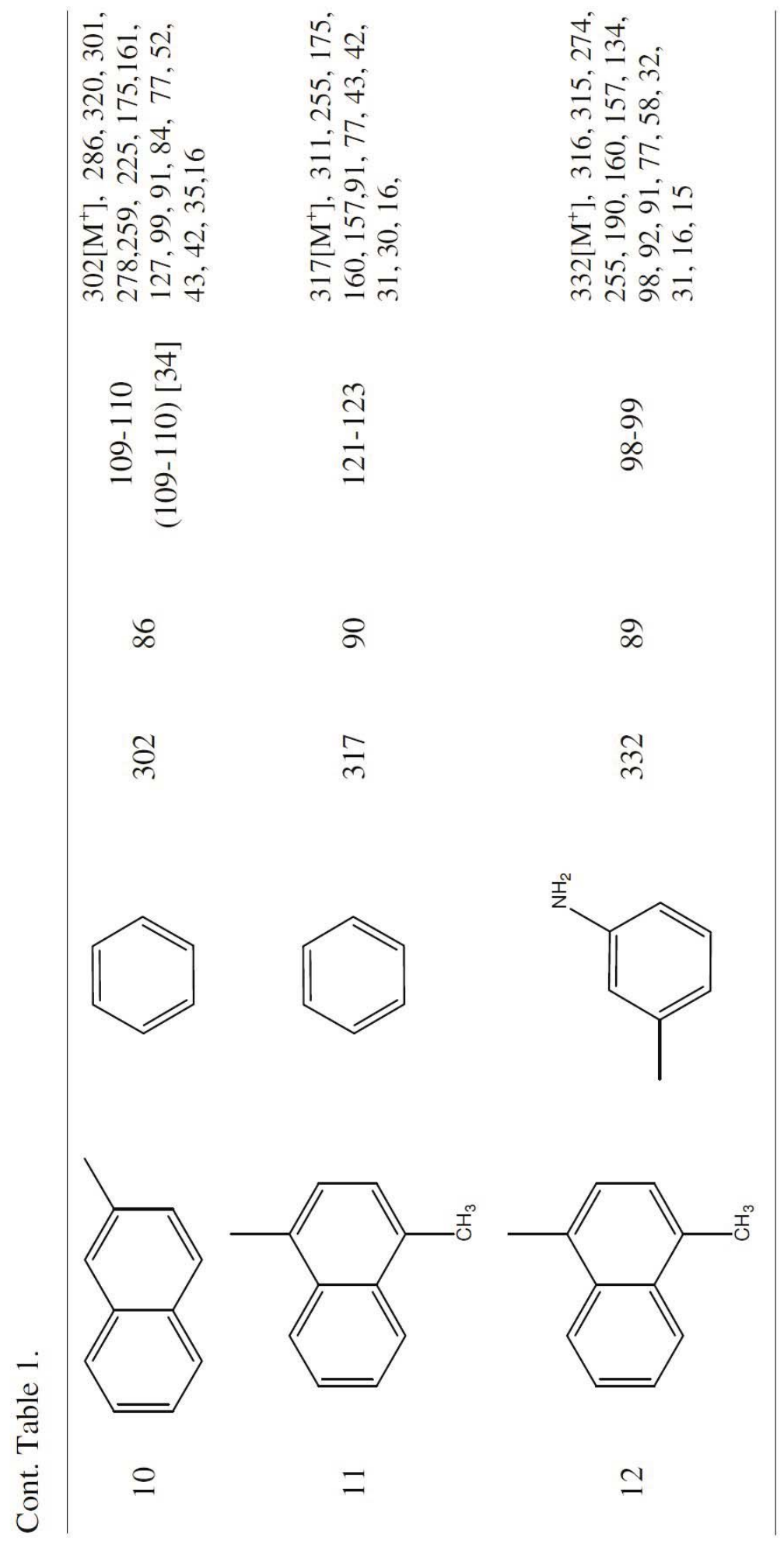




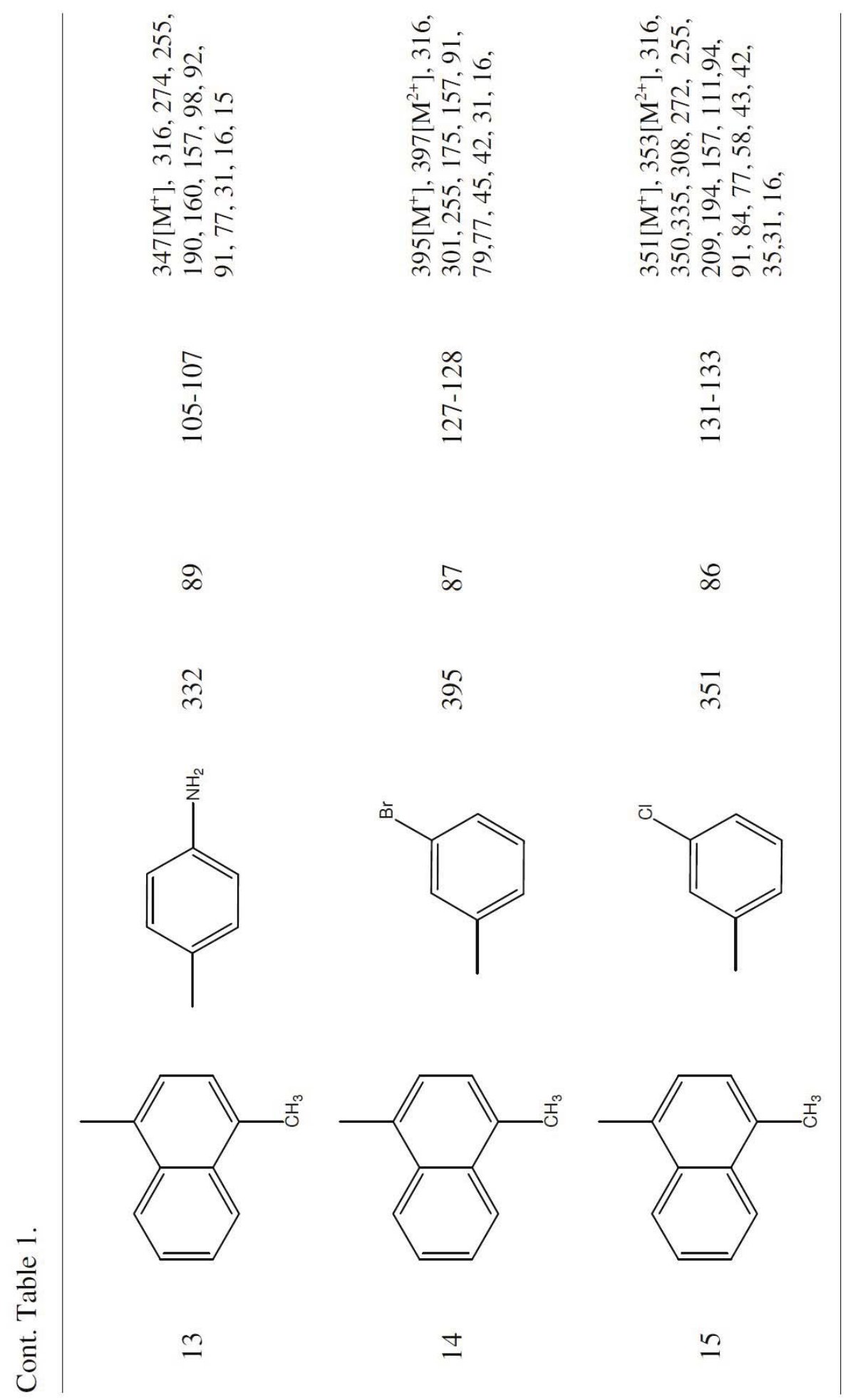




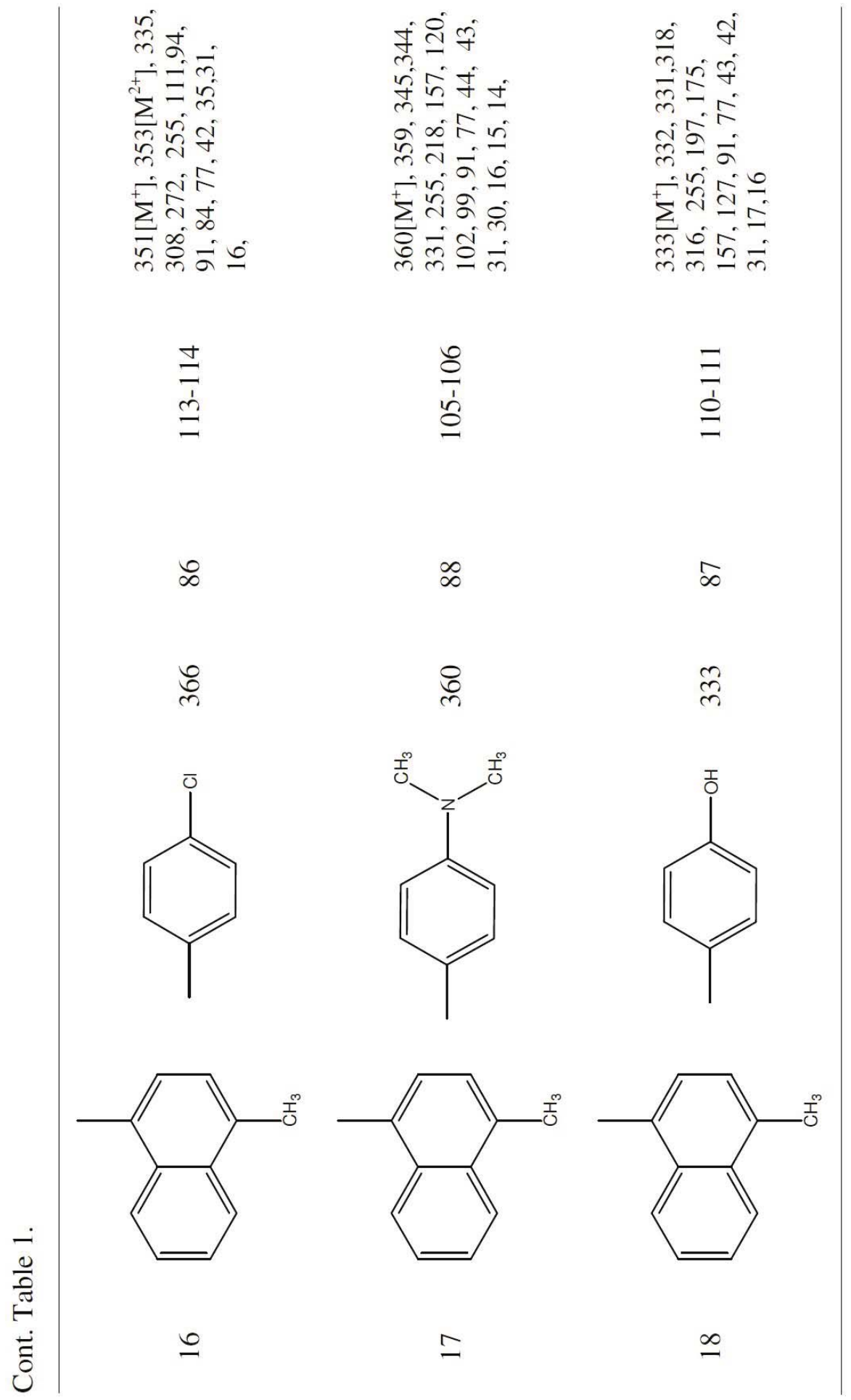




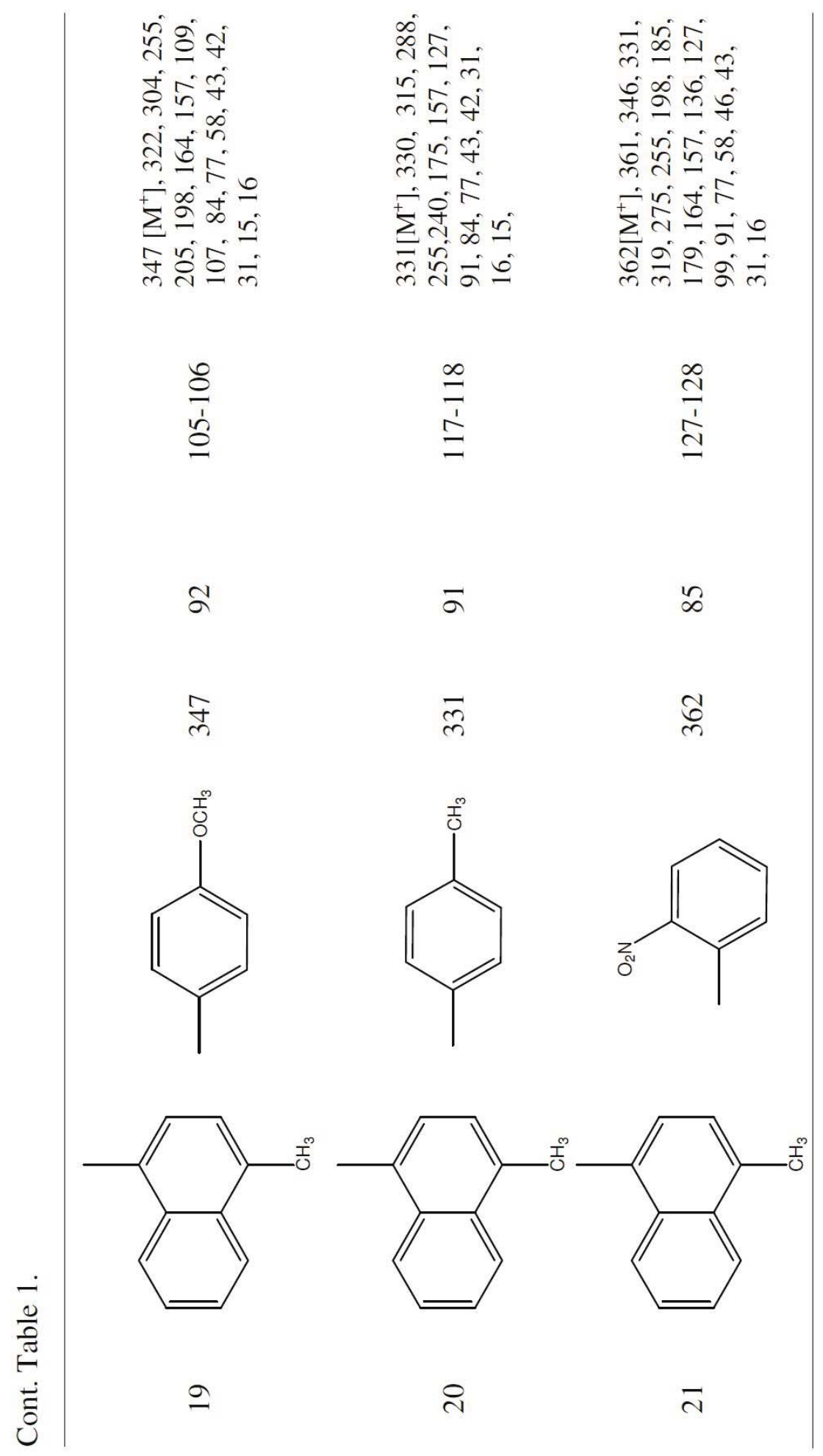




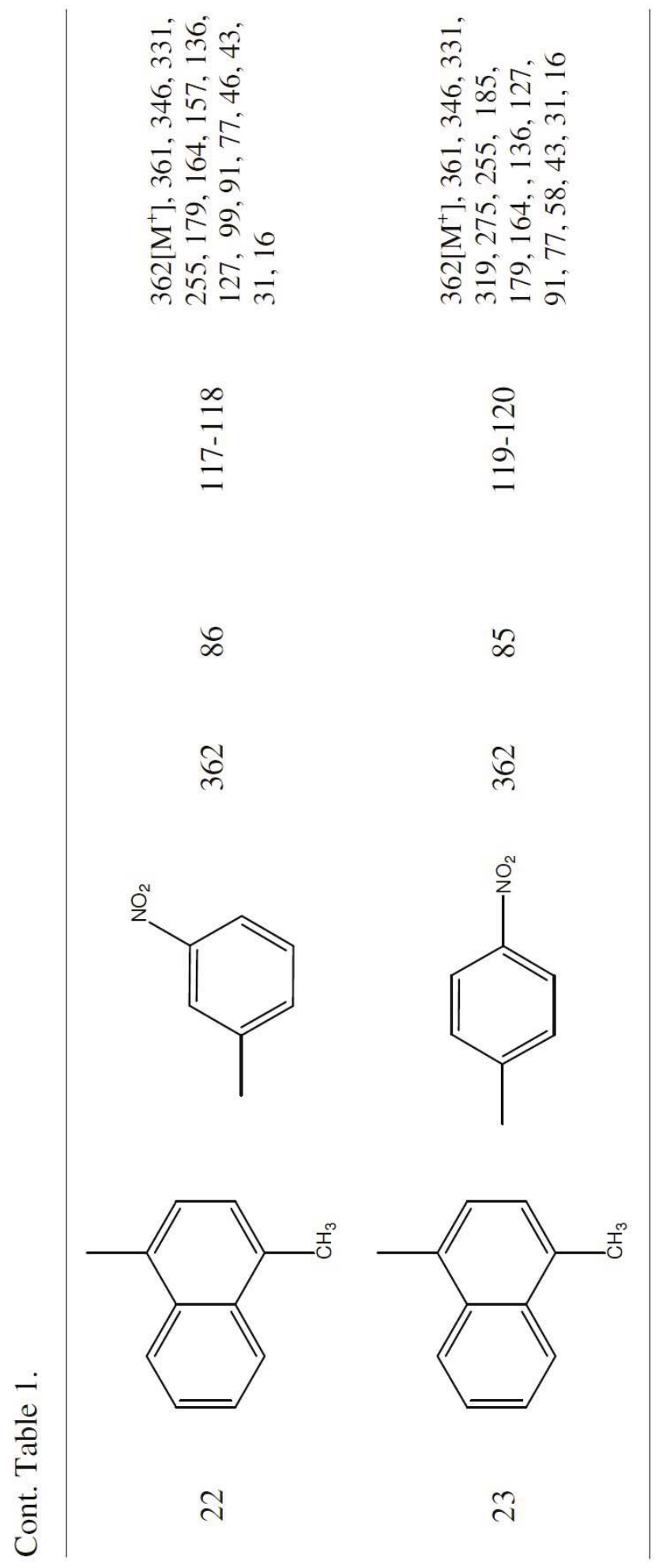


Table 2. The effect of solvents in conventional heating and without solvent in microwave irradiation on yield of 4-(4-Methyl-1-naphthyl)5,6-dihydro-6-phenyl-4H-1,3-oxazine-2-amine (entry 11).

\begin{tabular}{ccccc}
\hline \multicolumn{4}{c}{ Solvents } & \\
\cline { 1 - 3 } $\mathrm{MeOH}$ & EtOH & DCM & THF & Microwave irradiation \\
\cline { 1 - 4 } 61 & 60 & 64 & 65 & 90 \\
\hline
\end{tabular}

$\mathrm{MeOH}=$ Methanol; EtOH = Ethanol; DCM = Dihloromethane;

$\mathrm{THF}=$ Tetrahydrofuron .

\section{RESULTS AND DISCUSSION}

The author attempts made to synthesize some 4-aryl-5,6-dihydro6(substituted phenyl)-4H-1,3-oxazine-2-amine derivatives by cyclization of chalcones possess electron with-drawing as well as electron donating group as substituents, urea and in the presence of a catalyst hydroxylapatite using microwave irradiation. Hence the authors have synthesized some 4-aryl-5,6-dihydro-6(substituted phenyl)-4H-1,3-oxazine-2-amine derivatives by the cyclization of 2 mmole of chalcone, 2 mmole of urea under microwave irradiation with $0.2 \mathrm{~g}$ of hydroxyapatite catalyst at 550W for 4-6 minutes (Samsung Grill, GW73BD Microwave oven, 230V $\mathrm{A} / \mathrm{c}, 50 \mathrm{~Hz}, 2450 \mathrm{~Hz}, 100-750 \mathrm{~W}$ (IEC-705), (Scheme 1). During the course of this reaction hydroxyapatite catalyses cyclization between chalcones and urea followed by rearrangement gave the 1,3-oxazine amines. The yield of the oxazine in this reaction is more than $80 \%$. The chalcone containing electron donating substituent $\left(\mathrm{OCH}_{3}\right)$ gave higher yields than electron-withdrawing (halogens, $\mathrm{NO}_{2}$ ) substituents. Further we have investigated this cyclization reaction with equimolar quantities of the styryl 4-methyl-1-naphthyl ketone (entry 11) and urea under the same condition as above. In this reaction the obtained yield was $90 \%$. The effect of catalyst on this reaction was studied by varying the catalyst quantity from 0.05 to $0.2 \mathrm{~g}$. As the catalyst quantity is increased from 0.05 to $0.2 \mathrm{~g}$, the percentage of yield of product is increased from 84 to $90 \%$. About $1 \%$ product was obtained increased by increasing the amount of catalyst $0.5 \mathrm{~g}$. About $3 \%$ of product was obtained by increasing $0.15 \mathrm{~g}$ of catalyst. About $6 \%$ of product were obtained for increasing the amount of catalyst $0.2 \mathrm{~g}$. Further increase in the catalyst amount beyond 
$0.2 \mathrm{~g}$, there is no significant increase in the percentage of the product. The effect of catalyst loading is shown in Fig. 1.

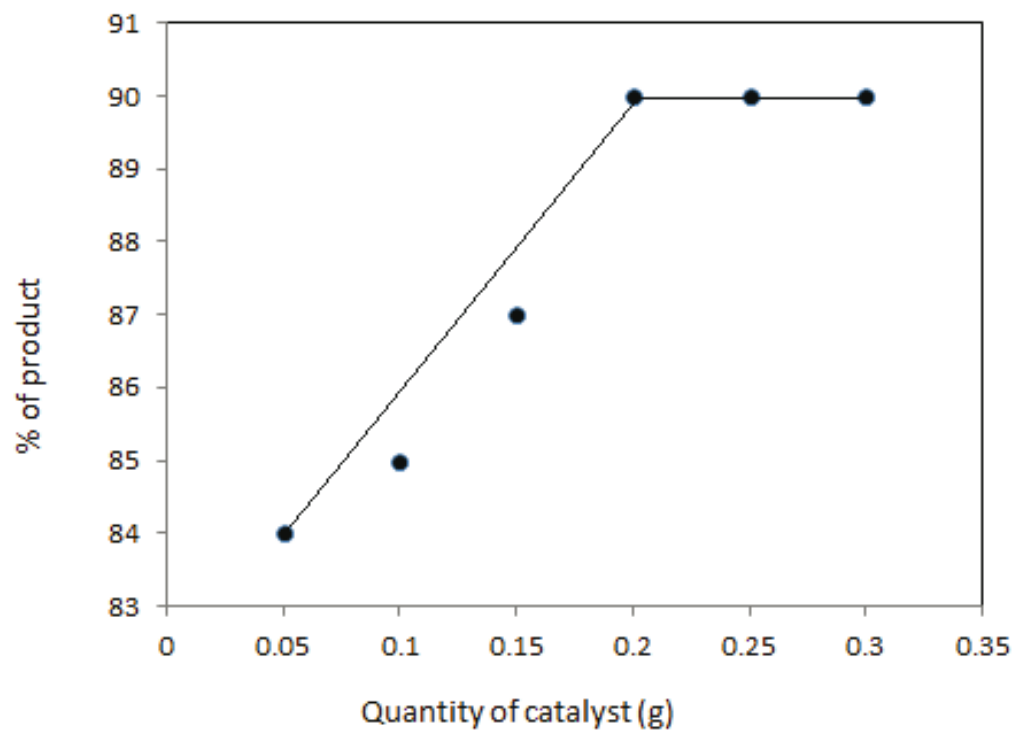

Fig. 1. Effect of catalyst loading.

The optimum quantity of catalyst loading was found to be $0.2 \mathrm{~g}$. The results, analytical and mass spectral data are summarized in Table 1. The effect of solvents on the yield was also studied with methanol, ethanol, dichloromethane and tetrahydrofuran from each component of the catalyst (entry 11). Similarly the effect of microwave irradiation was studied on each component of the catalyst. The effect of solvents on the yield of oxazine derivatives was presented in Table 2. From the table highest yield of oxazine obtained from the cyclization of chalcones and urea with the catalyst hydroxyapatite in microwave irradiation. The infrared and $\mathrm{nmr}$ spectroscopic data of selective oxazine-2-amine derivatives are summarized below.

4-(4-Methyl-1-naphthyl)-5,6-dihydro-6-phenyl-4H-1,3-oxazine-2-amine (11): FTIR(KBr): $353834(\mathrm{NH}), 1591(\mathrm{C}=\mathrm{N}), 1216(\mathrm{C}-\mathrm{O}-\mathrm{C}) \mathrm{cm}^{-1} .{ }^{1} \mathrm{H}$ NMR $\left(\mathrm{CDCl}_{3}-\mathrm{d}_{6}\right.$, TMS) $\delta: 2.331\left(\mathrm{~s}, 1 \mathrm{H}, \mathrm{NH}_{2}\right), 2.625\left(\mathrm{dd}, 1 \mathrm{H}, \mathrm{H}_{4}\right), 2.415\left(\mathrm{dd}, 1 \mathrm{H}, \mathrm{H}_{5}\right), 2.219(\mathrm{dd}, 1 \mathrm{H}$, $\left.\mathrm{H}_{5^{\prime}}\right), 4.232\left(\mathrm{dd}, 1 \mathrm{H}, \mathrm{H}_{6}\right), 4.572\left(\mathrm{~s}, 3 \mathrm{H}, \mathrm{OCH}_{3}\right), 6.511-7.338(\mathrm{~m}, 11 \mathrm{H}, \mathrm{Ar}-\mathrm{H}) \mathrm{ppm}$.; ${ }^{13} \mathrm{C}$ NMR $\left(\mathrm{CDCl}_{3}-\mathrm{d}_{6}, \mathrm{TMS}\right) \delta: 165.45\left(\mathrm{C}_{2}\right), 52.72\left(\mathrm{C}_{4}\right), 47.38\left(\mathrm{C}_{5}\right), 65.64\left(\mathrm{C}_{6}\right)$, 57.23 $\left(\mathrm{OCH}_{3}\right), 125.33-142.79(\mathrm{Ar}-\mathrm{C}) \mathrm{ppm}$. 
4-(4-Mthyl-1-naphthyl)-5,6-dihydro-6-(3-aminoyphenyl)-4H-1,3-oxazine-2-amine (12): $\operatorname{FTIR}(\mathrm{KBr})$ : $3560(\mathrm{NH}), 1615(\mathrm{C}=\mathrm{N}), 1224(\mathrm{C}-\mathrm{O}-\mathrm{C}) \mathrm{cm}^{-1} .{ }^{1} \mathrm{H}$ NMR $\left(\mathrm{CDCl}_{3}-\mathrm{d}_{6}, \mathrm{TMS}\right) \delta: 2.231\left(\mathrm{~s}, 1 \mathrm{H}, \mathrm{NH}_{2}\right), 2.528\left(\mathrm{dd}, 1 \mathrm{H}, \mathrm{H}_{4}\right), 2.458\left(\mathrm{dd}, 1 \mathrm{H}, \mathrm{H}_{5}\right)$, $2.233\left(\mathrm{dd}, 1 \mathrm{H}, \mathrm{H}_{5^{\prime}}\right), 4.358\left(\mathrm{dd}, 1 \mathrm{H}, \mathrm{H}_{6}\right), 3.238\left(\mathrm{~s}, 3 \mathrm{H}, \mathrm{CH}_{3}\right), 6.282-7.254(\mathrm{~m}, 10 \mathrm{H}$, Ar-H) ppm. ${ }^{13} \mathrm{C}$ NMR $\left(\mathrm{CDCl}_{3}-\mathrm{d}_{6}, \mathrm{TMS}\right) \delta: 164.38\left(\mathrm{C}_{2}\right), 51.09\left(\mathrm{C}_{4}\right), 47.79\left(\mathrm{C}_{5}\right)$, $66.31\left(\mathrm{C}_{6}\right), 28.12\left(\mathrm{CH}_{3}\right), 126.26-139.29(\mathrm{Ar}-\mathrm{C}) \mathrm{ppm}$.

4-(4-Methyl-1-naphthyl)-5,6-dihydro-6-(4-aminophenyl)-4H-1,3-oxazine-2-amine (13): FTIR $(\mathrm{KBr})$ : 3550(NH), 1615(C=N), 1226(C-O-C) $\mathrm{cm}^{-1} .{ }^{1} \mathrm{H}$ NMR $\left(\mathrm{CDCl}_{3}-\mathrm{d}_{6}, \mathrm{TMS}\right) \delta: 2.225\left(\mathrm{~s}, 1 \mathrm{H}, \mathrm{NH}_{2}\right), 2.532\left(\mathrm{dd}, 1 \mathrm{H}, \mathrm{H}_{4}\right), 2.469\left(\mathrm{dd}, 1 \mathrm{H}, \mathrm{H}_{5}\right)$, $2.246\left(\mathrm{dd}, 1 \mathrm{H}, \mathrm{H}_{5^{\prime}}\right), 4.378\left(\mathrm{dd}, 1 \mathrm{H}, \mathrm{H}_{6}\right), 3.228\left(\mathrm{~s}, 3 \mathrm{H}, \mathrm{CH}_{3}\right), 6.277-7.239(\mathrm{~m}, 10 \mathrm{H}$, Ar-H) ppm. ${ }^{13} \mathrm{C}$ NMR $\left(\mathrm{CDCl}_{3}-\mathrm{d}_{6}, \mathrm{TMS}\right) \delta: 164.76\left(\mathrm{C}_{2}\right), 51.49\left(\mathrm{C}_{4}\right), 47.61\left(\mathrm{C}_{5}\right)$, $66.90\left(\mathrm{C}_{6}\right), 27.14\left(\mathrm{CH}_{3}\right), 126.38-139.37(\mathrm{Ar}-\mathrm{C}) \mathrm{ppm}$.

4-(4-Methyl-1-naphthyl)-5,6-dihydro-6-(3-bromophenyl)-4H-1,3-oxazine-2-amine (14): FTIR(KBr): 3546(NH), $1622(\mathrm{C}=\mathrm{N}), 1219(\mathrm{C}-\mathrm{O}-\mathrm{C}) \mathrm{cm}^{-1} \cdot{ }^{1} \mathrm{H}$ NMR $\left(\mathrm{CDCl}_{3}-\mathrm{d}_{6}, \mathrm{TMS}\right) \delta: 2.217\left(\mathrm{~s}, 1 \mathrm{H}, \mathrm{NH}_{2}\right), 2.323\left(\mathrm{dd}, 1 \mathrm{H}, \mathrm{H}_{4}\right), 2.224\left(\mathrm{dd}, 1 \mathrm{H}, \mathrm{H}_{5}\right)$, $2.231\left(\mathrm{dd}, 1 \mathrm{H}, \mathrm{H}_{5^{\prime}}\right), 4.340\left(\mathrm{dd}, 1 \mathrm{H}, \mathrm{H}_{6}\right), 3.379\left(\mathrm{~s}, 3 \mathrm{H}, \mathrm{OCH}_{3}\right), 6.243-7.290(\mathrm{~m}$, $10 \mathrm{H}, \mathrm{Ar}-\mathrm{H}) \mathrm{ppm} .{ }^{13} \mathrm{C}$ NMR $\left(\mathrm{CDCl}_{3}-\mathrm{d}_{6}, \mathrm{TMS}\right) \delta: 164.49\left(\mathrm{C}_{2}\right), 51.80\left(\mathrm{C}_{4}\right)$, 47.30 $\left(\mathrm{C}_{5}\right), 66.67\left(\mathrm{C}_{6}\right), 25.32\left(\mathrm{CH}_{3}\right), 124.37-139.30(\mathrm{Ar}-\mathrm{C}) \mathrm{ppm}$.

4-(4-Methyl-1-naphthyl)-5,6-dihydro-6-(3-chlorophenyl)-4H-1,3-oxazine-2-amine (15): $\mathrm{FTIR}(\mathrm{KBr})$ : 3545(NH), 1613(C=N), 1222(C-O-C) $\mathrm{cm}^{-1} .{ }^{1} \mathrm{H}$ NMR $\left(\mathrm{CDCl}_{3}-\mathrm{d}_{6}, \mathrm{TMS}\right) \delta: 2.267\left(\mathrm{~s}, 1 \mathrm{H}, \mathrm{NH}_{2}\right), 2.329\left(\mathrm{dd}, 1 \mathrm{H}, \mathrm{H}_{4}\right), 2.421\left(\mathrm{dd}, 1 \mathrm{H}, \mathrm{H}_{5}\right)$, $2.256\left(\mathrm{dd}, 1 \mathrm{H}, \mathrm{H}_{5^{\prime}}\right), 4.329\left(\mathrm{dd}, 1 \mathrm{H}, \mathrm{H}_{6}\right), 3.247\left(\mathrm{~s}, 3 \mathrm{H}, \mathrm{CH}_{3}\right), 6.249-7.290(\mathrm{~m}, 10 \mathrm{H}$, $\mathrm{Ar}-\mathrm{H}) \mathrm{ppm} .{ }^{13} \mathrm{C}$ NMR $\left(\mathrm{CDCl}_{3}-\mathrm{d}_{6}, \mathrm{TMS}\right) \delta: 164.98\left(\mathrm{C}_{2}\right), 51.78\left(\mathrm{C}_{4}\right), \quad 47.09\left(\mathrm{C}_{5}\right)$, $66.36\left(\mathrm{C}_{6}\right), 21.23\left(\mathrm{CH}_{3}\right), 126.45-139.78(\mathrm{Ar}-\mathrm{C}) \mathrm{ppm}$.

4-(4-Methyl-1-naphthyl)-5,6-dihydro-6-(4-chlorophenyl)-4H-1,3-oxazine-2-amine (16): FTIR(KBr): $3555(\mathrm{NH}), 1615(\mathrm{C}=\mathrm{N}), 1230(\mathrm{C}-\mathrm{O}-\mathrm{C}) \mathrm{cm}^{-1} .{ }^{1} \mathrm{H}$ NMR $\left(\mathrm{CDCl}_{3}-\mathrm{d}_{6}, \mathrm{TMS}\right) \delta: 2.220\left(\mathrm{~s}, 1 \mathrm{H}, \mathrm{NH}_{2}\right), 2.319\left(\mathrm{dd}, 1 \mathrm{H}, \mathrm{H}_{4}\right), 2.418\left(\mathrm{dd}, 1 \mathrm{H}, \mathrm{H}_{5}\right)$, 2.229(dd, $\left.1 \mathrm{H}, \mathrm{H}_{5^{\prime}}\right), 4.367\left(\mathrm{dd}, 1 \mathrm{H}, \mathrm{H}_{6}\right), 3.232\left(\mathrm{~s}, 3 \mathrm{H}, \mathrm{CH}_{3}\right), 6.219-7.252(\mathrm{~m}, 10 \mathrm{H}$, Ar-H) ppm. ${ }^{13} \mathrm{C}$ NMR $\left(\mathrm{CDCl}_{3}-\mathrm{d}_{6}, \mathrm{TMS}\right) \delta: 164.23\left(\mathrm{C}_{2}\right), 51.22\left(\mathrm{C}_{4}\right), 47.43\left(\mathrm{C}_{5}\right)$, $66.78\left(\mathrm{C}_{6}\right), \quad 25.19\left(\mathrm{CH}_{3}\right), 126.26-139.67(\mathrm{Ar}-\mathrm{C}) \mathrm{ppm}$.

4-(4-Methyl-1-naphthyl)-5,6-dihydro-6-(4-dimethylaminophenyl)-4H-1,3-oxazine-2-amine (17): FTIR(KBr): 3530(NH), $1602(\mathrm{C}=\mathrm{N}), 1216(\mathrm{C}-\mathrm{O}-\mathrm{C}) \mathrm{cm}^{-1} \cdot{ }^{1} \mathrm{H}$ NMR $\left(\mathrm{CDCl}_{3}-\mathrm{d}_{6}, \mathrm{TMS}\right) \delta: 2.217\left(\mathrm{~s}, 1 \mathrm{H}, \mathrm{NH}_{2}\right), 2.332\left(\mathrm{dd}, 1 \mathrm{H}, \mathrm{H}_{4}\right), 2.424(\mathrm{dd}, 1 \mathrm{H}$, $\left.\mathrm{H}_{5}\right), 2.232\left(\mathrm{dd}, 1 \mathrm{H}, \mathrm{H}_{5^{\prime}}\right), 4.332\left(\mathrm{dd}, 1 \mathrm{H}, \mathrm{H}_{6}\right), 3.645\left(\mathrm{~s}, 6 \mathrm{H}, \mathrm{N}\left(\mathrm{CH}_{3}\right)_{2}\right), 3.233(\mathrm{~s}, 3 \mathrm{H}$, $\left.\mathrm{CH}_{3}\right), 26.234-7.278(\mathrm{~m}, 10 \mathrm{H}, \mathrm{Ar}-\mathrm{H}) \mathrm{ppm} .{ }^{13} \mathrm{C} \mathrm{NMR}\left(\mathrm{CDCl}_{3}-\mathrm{d}_{6}, \mathrm{TMS}\right) \delta: 164.89$ $\left(\mathrm{C}_{2}\right), 51.58\left(\mathrm{C}_{4}\right), 47.87\left(\mathrm{C}_{5}\right), 66.67\left(\mathrm{C}_{6}\right), \quad 44.89\left(\mathrm{~N}_{\left.\left(\mathrm{CH}_{3}\right)_{2}\right), 24.56\left(\mathrm{CH}_{3}\right), 126.22-}\right.$ 139.10(Ar-C)ppm.

4-(4-Methyl-1-naphthyl)-5,6-dihydro-6-(4-hydroxyphenyl)-4H-1,3-oxazine-2amine (18): FTIR(KBr): 3545(NH), 1611(C=N), $1212(\mathrm{C}-\mathrm{O}-\mathrm{C}) \mathrm{cm}^{-1} .{ }^{1} \mathrm{H}$ NMR $\left(\mathrm{CDCl}_{3}-\mathrm{d}_{6}, \mathrm{TMS}\right) \delta: 2.217\left(\mathrm{~s}, 1 \mathrm{H}, \mathrm{NH}_{2}\right), 2.343\left(\mathrm{dd}, 1 \mathrm{H}, \mathrm{H}_{4}\right), 2.476\left(\mathrm{dd}, 1 \mathrm{H}, \mathrm{H}_{5}\right)$, 2.233(dd, $\left.1 \mathrm{H}, \mathrm{H}_{5^{\prime}}\right), 4.358\left(\mathrm{dd}, 1 \mathrm{H}, \mathrm{H}_{6}\right), 3.268\left(\mathrm{~s}, 3 \mathrm{H}, \mathrm{CH}_{3}\right), 6.246-7.276(\mathrm{~m}, 10 \mathrm{H}$, 
Ar-H) ppm. ${ }^{13} \mathrm{C}$ NMR $\left(\mathrm{CDCl}_{3}-\mathrm{d}_{6}, \mathrm{TMS}\right) \delta: 164.6734\left(\mathrm{C}_{2}\right), 51.98\left(\mathrm{C}_{4}\right), 47.36\left(\mathrm{C}_{5}\right)$, $63.58\left(\mathrm{C}_{6}\right), 44.12\left(\mathrm{~N}\left(\mathrm{CH}_{3}\right)_{2}\right), 24.26\left(\mathrm{~s}, 3 \mathrm{H}, \mathrm{CH}_{3}\right), 126.65-139.98(\mathrm{Ar}-\mathrm{C}) \mathrm{ppm}$.

4-(4-Methyl-1-naphthyl)-5,6-dihydro-6-(4-methoxyphenyl)-4H-1,3-oxazine-2amine (19): $\mathrm{FTIR}(\mathrm{KBr})$ : 3545(NH), $1623(\mathrm{C}=\mathrm{N}), 1210(\mathrm{C}-\mathrm{O}-\mathrm{C}) \mathrm{cm}^{-1}$. ${ }^{1} \mathrm{H}$ NMR $\left(\mathrm{CDCl}_{3}-\mathrm{d}_{6}, \mathrm{TMS}\right) \delta: 2.214\left(\mathrm{~s}, 1 \mathrm{H}, \mathrm{NH}_{2}\right), 2.324\left(\mathrm{dd}, 1 \mathrm{H}, \mathrm{H}_{4}\right), 2.437\left(\mathrm{dd}, 1 \mathrm{H}, \mathrm{H}_{5}\right)$, 2.219(dd, $\left.1 \mathrm{H}, \mathrm{H}_{5^{\prime}}\right), 4.366\left(\mathrm{dd}, 1 \mathrm{H}, \mathrm{H}_{6}\right), 4.234\left(\mathrm{~s}, 3 \mathrm{H}, \mathrm{OCH}_{3}\right), 3.657\left(\mathrm{~s}, 3 \mathrm{H}, \mathrm{CH}_{3}\right)$, 6.267-7.289(m, 10H, Ar-H) ppm. ${ }^{13} \mathrm{C} \mathrm{NMR}\left(\mathrm{CDCl}_{3}-\mathrm{d}_{6}, \mathrm{TMS}\right) \delta: 164.57\left(\mathrm{C}_{2}\right)$, $51.67\left(\mathrm{C}_{4}\right), \quad 47.34\left(\mathrm{C}_{5}\right), 66.01\left(\mathrm{C}_{6}\right), \quad 64.34\left(\mathrm{~s}, 3 \mathrm{H}, \mathrm{OCH}_{3}\right), 24.64\left(\mathrm{~s}, 3 \mathrm{H}, \mathrm{CH}_{3}\right)$, 126.34-139.76(Ar-C)ppm.

4-(4-Methyl-1-naphthyl)-5,6-dihydro-6-(4-methylphenyl)-4H-1,3-oxazine-2-amine (20): FTIR(KBr): 3535(NH), 1612(C=N), 1211(C-O-C) $\mathrm{cm}^{-1} \cdot{ }^{1} \mathrm{H}$ NMR $\left(\mathrm{CDCl}_{3}-\mathrm{d}_{6}, \mathrm{TMS}\right) \delta: 2.215\left(\mathrm{~s}, 1 \mathrm{H}, \mathrm{NH}_{2}\right), 2.327\left(\mathrm{dd}, 1 \mathrm{H}, \mathrm{H}_{4}\right), 2.433\left(\mathrm{dd}, 1 \mathrm{H}, \mathrm{H}_{5}\right)$, 2.223(dd, $\left.1 \mathrm{H}, \mathrm{H}_{5^{\prime}}\right), 4.346\left(\mathrm{dd}, 1 \mathrm{H}, \mathrm{H}_{6}\right), 2.733$ (s, 3H, $\left.\mathrm{CH}_{3}\right), 4.213$ (s, 3H, $\mathrm{OCH}_{3}$ ), 6.223-7.216(m, 10H, Ar-H) ppm. ${ }^{13} \mathrm{C} \mathrm{NMR}\left(\mathrm{CDCl}_{3}-\mathrm{d}_{6}, \mathrm{TMS}\right) \delta: 164.26\left(\mathrm{C}_{2}\right)$, $\left.51.29\left(\mathrm{C}_{4}\right), 47.43 \mathrm{C}_{5}\right), 66.32\left(\mathrm{C}_{6}\right), 26.35\left(\mathrm{CH}_{3}\right), 64.66\left(\mathrm{OCH}_{3}\right), 114.33-145.26(\mathrm{Ar}-$ C)ppm.

4-(4-Methyl-1-naphthyl)-5,6-dihydro-6-(2-nitrophenyl)-4H-1,3-oxazine-2-amine (21): FTIR(KBr): 3556(NH), $1625(\mathrm{C}=\mathrm{N}), 1214(\mathrm{C}-\mathrm{O}-\mathrm{C}) \mathrm{cm}^{-1}$. ${ }^{1} \mathrm{H}$ NMR $\left(\mathrm{CDCl}_{3}-\mathrm{d}_{6}, \mathrm{TMS}\right) \delta: 2.218\left(\mathrm{~s}, 1 \mathrm{H}, \mathrm{NH}_{2}\right), 2.335\left(\mathrm{dd}, 1 \mathrm{H}, \mathrm{H}_{4}\right), 2.446\left(\mathrm{dd}, 1 \mathrm{H}, \mathrm{H}_{5}\right)$, 2.227(dd, $\left.1 \mathrm{H}, \mathrm{H}_{5^{\prime}}\right), 4.348\left(\mathrm{dd}, 1 \mathrm{H}, \mathrm{H}_{6}\right), 3.268\left(\mathrm{~s}, 3 \mathrm{H}, \mathrm{CH}_{3}\right), 6.233-7.267(\mathrm{~m}, 10 \mathrm{H}$, Ar-H) ppm. ${ }^{13} \mathrm{C}$ NMR $\left(\mathrm{CDCl}_{3}-\mathrm{d}_{6}, \mathrm{TMS}\right) \delta: 64.90\left(\mathrm{C}_{2}\right), 51.31\left(\mathrm{C}_{4}\right), \quad 47.34\left(\mathrm{C}_{5}\right)$, $66.76\left(\mathrm{C}_{6}\right), \quad 27.87\left(\mathrm{~s}, 3 \mathrm{H}, \mathrm{CH}_{3}\right), 114.45-145.67(\mathrm{Ar}-\mathrm{C}) \mathrm{ppm}$.

4-(4-Methyl-1-naphthyl)-5,6-dihydro-6-(3-nitrophenyl)-4H-1,3-oxazine-2-amine (22): FTIR(KBr): 3555(NH), $1618(\mathrm{C}=\mathrm{N}), 1223(\mathrm{C}-\mathrm{O}-\mathrm{C}) \mathrm{cm}^{-1}$. ${ }^{1} \mathrm{H}$ NMR $\left(\mathrm{CDCl}_{3}-\mathrm{d}_{6}, \mathrm{TMS}\right) \delta: 2.215\left(\mathrm{~s}, 1 \mathrm{H}, \mathrm{NH}_{2}\right), 2.338\left(\mathrm{dd}, 1 \mathrm{H}, \mathrm{H}_{4}\right), 2.460\left(\mathrm{dd}, 1 \mathrm{H}, \mathrm{H}_{5}\right)$, 2.234(dd, $\left.1 \mathrm{H}, \mathrm{H}_{5^{\prime}}\right), 4.365\left(\mathrm{dd}, 1 \mathrm{H}, \mathrm{H}_{6}\right), 3.356\left(\mathrm{~s}, 3 \mathrm{H}, \mathrm{CH}_{3}\right), 6.546-7.289(\mathrm{~m}, 10 \mathrm{H}$, Ar-H) ppm. ${ }^{13} \mathrm{C}$ NMR $\left(\mathrm{CDCl}_{3}-\mathrm{d}_{6}, \mathrm{TMS}\right) \delta: 164.78\left(\mathrm{C}_{2}\right), 51.34\left(\mathrm{C}_{4}\right), 47.42\left(\mathrm{C}_{5}\right)$, $66.67\left(\mathrm{C}_{6}\right), \quad 24.67\left(\mathrm{~s}, 3 \mathrm{H}, \mathrm{CH}_{3}\right), 124.98-145.47(\mathrm{Ar}-\mathrm{C}) \mathrm{ppm}$.

4-(4-Methyl-1-naphthyl)-5,6-dihydro-6-(4-nitrophenyl)-4H-1,3-oxazine-2-amine (23): FTIR(KBr): 3550(NH), $1625(\mathrm{C}=\mathrm{N}), 1230(\mathrm{C}-\mathrm{O}-\mathrm{C}) \mathrm{cm}^{-1}$. ${ }^{1} \mathrm{H}$ NMR $\left(\mathrm{CDCl}_{3}-\mathrm{d}_{6}, \mathrm{TMS}\right) \delta: 2.215\left(\mathrm{~s}, 1 \mathrm{H}, \mathrm{NH}_{2}\right), 2.333\left(\mathrm{dd}, 1 \mathrm{H}, \mathrm{H}_{4}\right), 2.454\left(\mathrm{dd}, 1 \mathrm{H}, \mathrm{H}_{5}\right)$, 2.236(dd, $\left.1 \mathrm{H}, \mathrm{H}_{5^{\prime}}\right), 4.343\left(\mathrm{dd}, 1 \mathrm{H}, \mathrm{H}_{6}\right), 3.223\left(\mathrm{~s}, 3 \mathrm{H}, \mathrm{CH}_{3}\right), 6.534-7.234(\mathrm{~m}, 10 \mathrm{H}$, Ar-H) ppm. ${ }^{13} \mathrm{C}$ NMR $\left(\mathrm{CDCl}_{3}-\mathrm{d}_{6}, \mathrm{TMS}\right) \delta: 164.34\left(\mathrm{C}_{2}\right), 51.65\left(\mathrm{C}_{4}\right), 47.37\left(\mathrm{C}_{5}\right)$, $66.56\left(\mathrm{C}_{6}\right), 24.64\left(\mathrm{~s}, 3 \mathrm{H}, \mathrm{CH}_{3}\right), 124.46-145.43(\mathrm{Ar}-\mathrm{C}) \mathrm{ppm}$.

\section{CONCLUSIONS}

Some unsaturated 4-aryl-5,6-dihydro-6(substituted phenyl)-4H-1,3oxazine-2-amines derivatives including 4-(4-methyl-1-naphthyl)-5,6dihydro-6-(substituted phenyl)-oxazine-2-amines have been synthesised 
by solvent-free cyclization of aryl chalcones and urea in presence of hydroxy apatite catalyst under microwave irradiation. The yields of these oxazines were more than $85 \%$. This synthetic methodology offers nonhazardous, solvent-free cyclization, easy-workup procedure, shorter reaction time and better yields. These oxazine derivatives were characterized by their physical constants, spectral data and these data supports the compounds.

\section{REFERENCES}

[1] J. Cherian, I. Choi, A. Nayyar, U.H. Manjunatha, T. Mukherjee, Y.S. Lee, H.I. Boshoff, R. Singh,Y. H. Ha, M. Goodwin, S.B. Lakshminarayana, P. Niyomrattanakit, J. Jiricek, R. Ravindran, T. Dick, T.H. Keller, V. Dartois, C.E. Barry, J. Med. Chem., 54(16), 5639 (2011).

[2] A.M. Thompson, H.S. Sutherl , D.B. Palmer, I. Kmentova, A. Blaser, S.G. Franzblau, B. Wan, Y. Wang, Z. Ma, W.A. Denny, J. Med. Chem., 54(16), 6563, (2011).

[3] I.P. Yakovlev, A.V. Prep'yalov, B.A. Ivin, Chem. Heterocycl. Compd, 30(3), 255, (1994).

[4] M.K. Manjula, K.M.L. Rai, S.L. Gaonkar, K.A. Raveesha S. Satish, Eur. J. Med. Chem., 44, 280 (2009).

[5] B.P. Mathew, A. Kumar, S. Sharma, P.K. Shukla, M. Nath, Eur. J. Med. Chem. 45, 1502, (2010).

[6] M.J. Elarfi, H.A. Al-Difar, Sci. Rev.Chem. Commun., 2(2), 103, (2012).

[7] V. Tiwari, J. Meshram, P. Ali, J. Sheikh U. Tripathi, J. Enzyme Inhibit.Med. Chem., 26(4), 569, (2011).

[8] B.C. Das, A.V. Madhukumar, J. Anguiano S. Mani, Bioorg. Med. Chem. Lett., 19(15), 4204, (2009).

[9] D. Zhou, B.L. Harrison, U. Shah, T.H. Andree, G.A. Hornby, R. Scerni, Bioorg. Med. Chem. Lett., 16(5), 1338,(2006).

[10] S. Wang, Y. Li, Y. Liu, A. Lu Q. You, Bioorg. Med. Chem. Lett., 18(14), 4095, (2008).

[11] Y. Ando, K. Ando, M. Yamaguchi, J. Kunitomo, M. Koida, R. Fukuyama, Bioorg. Med. Chem. Lett., 16(22), 5849, (2006).

[12] L. Benameur, Z. Bouaziz, P. Nebois, M. H. Bartoli, M. Boitard, H. Fillion, Chem. Pharm. Bulletin (Tokyo), 44(3), 605, (1996). 
[13] K. Roy, I. Mitra A. Saha, Chem. Biol. Drug Design, 74(5), 507, (2009)..

[14] A. Blaser, D. Palmer, S. H. Sutherl , I. Kmentova, S. G. Franzblau B. Wan, J. Med.Chem., 55(1), 312, (2012).

[15] L. Seal, D. Von Hoff. R. Lawrence, E. Izbicka R.M. Jamison, Investi. New Drugs, 15(4), 289, (1997).

[16] B. Brudeli, L.R. Moltzau, K.W. Andressen, K.A. Krobert, J. Klaveness F.O. Levy, Bioorg. Med. Chem., 18(24), 8600, (2010).

[17] M. Akhter, A. Husain, N. Akhter M.S.Y. Khan, Indian J. Pharm. Sci., 73, 101, (2011).

[18] D. Gothi, J.M. Joshi, Recent Patent. Antiinfect. Drug Discov., 6(1), 27, (2011).

[19] K.S. Oh. S. Lee, J.K. Choi B.H. Lee, Comb. Chem. Higher Throughput Screen, 13(9), 790 (2010).

[20] S.Y. Cho, J.Y. Baek, S.S. Han. S.K. Kang, J.D. Ha J.H. Ahn, Bioorg. Med. Chem. Lett., 16(3), 499, (2006).

[21] S.F. Lee, Q. Vérolet A. Fürstenberg, Angew. Chem. Int. Ed., 52(34), 8948, (2013).

[22] L.A. Kass, Biotech Histochem, 70(1), 19, (1995).

[23] L.A. Kass, Biotech Histochem, 70(1), 29,(1995).

[24] C. Jung, B.K. Müller, D.C. Lamb, F. Nolde, K. Müllen C. Bräuchle, J. Am.Chem. Soc., 128(15), 5238, (2006).

[25] A.K. Verma, D. Chioudhary, R.K. Saunthwal, V. Rustagi, M. Patel R.K. Tiwari, J. Org. Chem., 78(13), 6657, (2013).

[26] M.A. Khalilzadeh, I. Yavari, Z. Hossaini H. Sadeghifar, Monatsch Chem., 140, 467, (2009).

[27] S.B. Sapkal, K.F. Shelke, A.H. Kategaonkar M.S. Shingare, Green Chem. Lett., 2(2), 57, (2009).

[28] J.H. MacMillan S.S. Washburne, Detailed Synthetic Procedure for 4-(4-bromophenyl)-1,3(3H) Oxazine-2,6-Dione related 4 5-aryl substituted -1,3(3H) Oxazine-2,6-Diones. Spectroscopic analytical data are included. Temple University, http://www.archive.org. (2013).

[29] G. Thirunarayanan, P. Mayavel K. Thirumurthy, Spectrochim. Acta Part A, 91, 18, (2012).

[30] G. Thirunarayanan, S. Surya, S. Srinivasan, G. Vanangamudi, V. Sathiyendiran, Spectrochim. Acta Part A, 75, 152, (2010).

[31] M. Subramanian, G. Vanangamudi G. Thirunarayanan, Spectrochim. Acta Part A, 110, 116, (2013). 
[32] Z. Turgut, E. Pelit A. Koycil, Molecules, 12, 345, (2007).

[33] M. Ansari, S. M. Naghib, F. Moztarzadeh A. Salati, CeramicsSilikáty, 55, 123, (2011).

[34] G. Thirunarayanan, Int. J. Sci. Res. Know, 1, 580, (2013). 